\section{Actividad física y tiempo sedente se asocian a sospecha de deterioro cognitivo en población adulta mayor chilena}

\author{
FELIPE POBLETE-VALDERRAMA ${ }^{1,16, a *}$, \\ CAROL FLORES RIVERA ${ }^{2, a *}$, FANNY PETERMANN-ROCHA ${ }^{3, b}$, \\ ANA MARÍA LEIVA ${ }^{4, c}$, MARÍA ADELA MARTÍNEZ- \\ SANGUINETTI $^{5, \mathrm{c}}$, CLAUDIA TRONCOSO $^{6, \mathrm{~b}}$, \\ LORENA MARDONES ${ }^{7, c}$, MARCELO VILLAGRÁN ${ }^{7, c}$, \\ GABRIELA NAZAR ${ }^{8, \mathrm{~d}}$, NATALIA ULLOA ${ }^{9, \mathrm{c}}$, \\ MIQUEL MARTORELL ${ }^{10, \mathrm{~b}}$, XIMENA DÍAZ-MARTÍNEZ $^{11, \mathrm{a}}$, \\ FABIÁN LANUZA ${ }^{12, b}$, ALEX GARRIDO-MÉNDEZ ${ }^{13, a}$, \\ CARLOS CELIS-MORALES ${ }^{3,14,15, a}$; en representación del Grupo \\ de Investigación ELHOC
}

\section{Physical activity and sedentary behaviours are associated with cognitive impairment in Chilean older adults}

Background: Lifestyle factors could promote healthy ageing. Aim: To investigate the association between physical activity (PA), sedentary behavior and cognitive impairment in Chilean older adults. Material and Methods: We included 1,390 participants from the National Health Survey (2009-2010). The Mini-Mental State Examination was used to diagnose cognitive impairment. Physical activity and sedentary behavior were assessed with the Global Physical Activity Questionnaire (GPAQ). Logistic regression was performed to investigate the associations. Results: Compared with older adults with lower levels of $P A(<48 \mathrm{~min} /$ day $)$, those with middle (48-248 min/day) and higher (>248 min/day) levels of $P A$ had lower odds for cognitive impairment (Odds ratio (OR): 0.57 [95\% confidence intervals (CI): $0.33 ; 0.82$ ], $p<0.01$ and 0.58 [95\% CI: 0.32; 0.83], $p<0.01$, respectively). Participants who reported spending more than 8 hours/day sitting had a high odds for cognitive impairment compared to those who spent $<4$ hours/ day (OR: 3.70 [95\% CI: 1.37; 6.03], $p=0.01$ ). Conclusions: Both $P A$ and sedentary behavior were independently associated with cognitive decline independent of major confounding factors in Chilean older adults.

(Rev Med Chile 2019; 147: 1247-1255)

Key words: Aging; Cognitive Impairment; Physical Activity, Sedentary Behaviours.
'Escuela de Kinesiología, Facultad de Salud, Universidad Santo Tomás, Sede Valdivia. Valdivia, Chile.

2Escuela de Salud Pública, Facultad de Medicina, Programa de Doctorado en Salud Pública, Santiago. Chile.

${ }^{3}$ Institute of Health and wellbeing, University of Glasgow, Glasgow, United Kingdom.

${ }^{4}$ Instituto de Anatomía, Histología y Patología, Facultad de Medicina, Universidad Austral de Chile. Valdivia, Chile.

${ }^{5}$ Instituto de Farmacia, Facultad de Ciencias, Universidad Austral de Chile. Valdivia, Chile. ${ }^{6}$ CIEDE-UCSC, Facultad de Medicina, Universidad Católica de la Santísima Concepción. Concepción, Chile.

${ }^{7}$ Departamento de Ciencias Básicas. Universidad Católica de la Santísima Concepción. Concepción, Chile.

${ }^{8}$ Departamento de Psicología y Centro Vida Saludable, Universidad de Concepción. Concepción, Chile.

${ }^{9}$ Departamento de Bioquímica Clínica e Inmunología, Facultad de Farmacia y Centro de Vida Saludable de la Universidad de Concepción. Concepción, Chile.

${ }^{10}$ Departamento de Nutrición y Dietética. Facultad de Farmacia, Universidad de Concepción.

Concepción, Chile.

${ }^{11}$ Grupo de Investigación Calidad de Vida, Departamento Ciencias de la Educación, Universidad del Biobío. Chillán, Chile.

${ }^{12}$ Departamento Pediatría y Cirugía Infantil, Facultad de Medicina, Universidad de La Frontera. Temuco, Chile.

${ }^{13}$ Departamento de Ciencias del Deporte y Acondicionamiento Físico, Universidad Católica de la Santísima Concepción. Concepción, Chile. ${ }^{14} \mathrm{BHF}$ Glasgow Cardiovascular Research Centre, Institute of Cardiovascular and Medical Science, University of Glasgow, Glasgow, United Kingdom. ${ }^{15}$ Centro de Investigación en Fisiología del Ejercicio-CIFE, Universidad Mayor. Santiago, Chile. ${ }^{16}$ Facultad de Educación, Universidad Católica de la Santísima Concepción. Concepción, Chile. aprofesor Educación Física.

${ }^{\mathrm{b}}$ Nutricionista.

'Bioquímico.

dPsicóloga.

Trabajo no recibió financiamiento.

Los autores declaran no tener conflictos de interés. *estos autores contribuyeron de igual forma y son considerados como primer primer autor compartido.

Recibido el 7 de octubre de 2018, aceptado el 10 de septiembre de 2019.

Correspondencia a:

Dr. Carlos Celis-Morales

Glasgow Cardiovascular Research Centre Institute of Cardiovascular and Medical Sciences. University of Glasgow.

G12 8TA, Glasgow. United Kingdom.

Carlos.Celis@glasgow.ac.uk 
Pistan ara el año 2050 la Organización Mundial de la Salud (OMS) proyecta que el crecimiento de la población adulta mayor (mayores de 60 años) a nivel mundial aumentará de 12\% a $22 \%$, lo que gatillará que una quinta parte de la población se encuentre en este grupo etario, sobrepasando los 2.000 millones de personas ${ }^{1,2}$. El aumento de la esperanza de vida vinculada al incremento en la población adulta mayor, implicará un cambio en el perfil epidemiológico de este grupo etario, caracterizado por un aumento de la prevalencia de multimorbilidad ${ }^{3,4}$ como así también un aumento en la prevalencia de deterioro cognitivo y patologías asociadas, como Alzheimer y demencia ${ }^{5}$.

La adopción de estilos de vida saludable, la práctica regular de actividad física $(\mathrm{AF})$, bajos niveles de tiempo sedente (tiempo destinado a estar sentado), han sido identificados como factores protectores contra el deterioro cognitivo en población europea y americana ${ }^{6-10}$. Sin embargo, la evidencia a nivel nacional es escasa ${ }^{10,11}$. Considerando que existe evidencia que siguiere que deterioro cognitivo se asocia a AF y tiempo sedente $^{6-10}$, es importante considerar cual es el escenario para la población adulta mayor en Chile. El 35,9\% de la población chilena destina más de 4 h al día a estar sentado y que $27,1 \%$ es físicamente inactiva (realiza menos de $150 \mathrm{~min}$ de AF de intensidad moderada o su equivalente a la semana $)^{12}$. No obstante, esta última cifra aumenta drásticamente con la edad, llegando a 63\% y $56 \%$ de inactividad física en mujeres y hombres mayores a 80 años, respectivamente ${ }^{13}$. Teniendo en cuenta estos antecedentes, la inactividad física y tiempo sedente podrían ser importantes factores de riesgo para sospecha de deterioro cognitivo en población adulta mayor en Chile; sin embargo, se desconoce su actual asociación. Por ende, el objetivo de este estudio fue investigar como la práctica de AF y tiempo sedente se asocian con sospecha de deterioro cognitivo en población adulta mayor chilena.

\section{Materiales y Métodos}

\section{Diseño del estudio}

Estudio de corte transversal que incorpora análisis secundarios de 1.384 participantes pertenecientes a población adulta mayor (según los criterios de la OMS (personas $>60$ años $^{14}$ ) de la Encuesta Nacional de salud 2009-2010 (ENS 2009-2010).

La ENS 2009-2010 corresponde a un estudio de prevalencia realizado en hogares en una muestra nacional, probabilística, estratificada y multietápica de 5.412 personas mayores de 15 años con representatividad nacional, regional, $y$ área urbana/rural. Sin embargo, con el objetivo de expandir la muestra a nivel nacional se utilizaron factores de expansión sugeridos por la ENS, por lo cual los datos presentados en este estudio representan a 2.392.764 de la población adulta mayor en Chile.

El protocolo de estudio fue aprobado por el Comité de Ética de la Escuela de Medicina de la Pontificia Universidad Católica de Chile. Todos los participantes firmaron un consentimiento informado ${ }^{15}$.

Sospecha de deterioro cognitivo se determinó mediante la aplicación del cuestionario Minimental (MMSE, por sus siglas en inglés), versión abreviada $^{15,16}$. La versión abreviada consta de 6 preguntas, con un puntaje máximo de 19 puntos, siendo el punto de corte utilizado para determinar sospecha mayor o igual a 13 puntos ${ }^{17}$.

Para determinar las variables de hábito tabáquico, se consideraron preguntas basadas en el instrumento mínimo de vigilancia de tabaquismo, utilizado por Organización Panamericana de la Salud ${ }^{15}$. Las variables demográficas como edad, sexo, zona geográfica de residencia (urbano/rural), nivel de escolaridad (básica $<8$ años, media 8 a 12 años, y educación superior $>12$ años) e ingreso económico (Bajo < 250.000; Medio 250.000 a 450.000; Alto $>450.000$ pesos chilenos) fueron recolectadas mediante el uso de cuestionarios validados en población nacional ${ }^{15}$. El tiempo promedio destinado a dormir en un día normal y existencias de enfermedades crónicas no transmisibles (diabetes mellitus tipo 2 [DMT2], hipertensión arterial [HTA], depresión y problemas auditivos) fueron recolectadas mediante cuestionarios de auto-reporte ${ }^{15}$.

El estado nutricional se clasificó según el índice de masa corporal (IMC) de acuerdo a las recomendaciones del MINSAL para población adulta mayor: bajo peso: $<22,9 \mathrm{~kg} / \mathrm{m}^{2}$; normo peso: 23,0 - 27,9 $\mathrm{kg} / \mathrm{m}^{2}$; sobrepeso: $28,0-31,9 \mathrm{~kg} / \mathrm{m}^{2}$ y obesidad: $\geq 32,0 \mathrm{~kg} / \mathrm{m}^{218}$, respecto a la obesidad central, ésta se definió por un perímetro de cin- 
tura $(\mathrm{PC}) \geq 88 \mathrm{~cm}$ para mujeres $\mathrm{y} \geq 102 \mathrm{~cm}$ para hombres, según los puntos de corte sugeridos por la $\mathrm{OMS}^{15,19}$.

Los niveles de AF, el tiempo destinado a las actividades de transporte, y las actividades de intensidad moderada o vigorosa de la población, fueron determinados con el cuestionario Global Physical Activity Questionnaire versión 2 (GPAQ v2 $)^{20}$, el cual ha sido validado internacionalmente y en población latina. La AF total es presentada como la suma del tiempo reportado en actividades de transporte, de intensidad moderada y vigorosa, tanto en el trabajo como en el tiempo libre. Esta variable fue expresada en METs/min/ semana, para considerar las diferencias en gasto energético asociada a cada una de las actividades. Se consideró como punto de corte para inactividad física un gasto energético menor a $600 \mathrm{METs} / \mathrm{min} /$ semana. Los niveles de tiempo sedente fueron determinados mediante el auto-reporte de tiempo destinado a actividades que involucren estar sentado o reclinado durante el tiempo libre o de trabajo (por ejemplo, tiempo sentado frente al computador o TV, viajando en bus, tren o auto) a través del mismo cuestionario GPAQ v2.

El índice de alimentación saludable fue estimado en base a la metodología descrita por Dussaillant ${ }^{21,22}$. Se obtuvo información con respecto al consumo de pescados, frutas, verduras, cereales integrales y lácteos (principalmente orientado a detectar intolerancia a la lactosa). Adicionalmente, se calculó un índice de dieta saludable (IDS), el cual fue construido integrando la información de consumo de todos los alimentos encuestados, exceptuando a los lácteos, ya que la encuesta no hizo distinción entre lácteos enteros y descremados. El IDS se calculó como la suma de los puntajes asignados a los 4 grupos de alimentos, quedando con un rango posible de puntaje entre 0 a 4 puntos.

\section{Análisis estadístico}

Todos los análisis se realizaron utilizando los factores de expansión de la ENS 2009-2010 aplicados en el programa STATA MP versión 15. Los datos de caracterización de la población estudiada son presentados como promedio para variables continuas, y como porcentaje para variables categóricas, ambos fueron expandidos a población nacional con sus respectivos $95 \%$ de intervalos de confianza (95\% IC).
Para investigar la asociación de sospecha de deterioro cognitivo según niveles de $\mathrm{AF}$ y niveles de tiempo sedente, las variables de exposición (AF y tiempo sedente) fueron transformadas a tertiles (bajo, medio y alto). Los puntos de corte para AF fueron: bajo $<48$ (grupo de referencia), medio $48-248$ y alto $>248$ min por día. Para tiempo sedente se utilizaron los siguientes puntos de corte: bajo $<4$ (grupo de referencia), medio $4-8$, y alto $>8 \mathrm{~h}$ por día. Se utilizaron análisis de regresión logística para investigar la asociación con sospecha de deterioro cognitivo. Los resultados son presentados como odds ratio (OR) y sus respectivos $95 \%$ intervalos de confianza (95\% IC).

Todos los análisis fueron ajustados por variables de confusión tomando en cuenta como éstas se asocian tanto a los factores de exposición como a la variable de salida (sospecha de deterioro cognitivo). La inclusión de estas variables se realizó progresivamente mediante la utilización de cuatro modelos estadísticos. El Modelo 0 no ajustado; Modelo 1 ajustado por edad, sexo, región, zona geográfica, nivel educacional e ingreso económico; Modelo 2 ajustado por el Modelo 1 más IMC, tabaquismo, índice de alimentación saludable, horas destinadas a dormir, AF o tiempo sedente, solo cuando estos no fueron incluidos como variable de exposición; Modelo 3 fue ajustado por el Modelo 2 más DMT2, HTA, problemas auditivos y diagnóstico de depresión. El nivel de significancia fue definido como $\mathrm{p}<0,05$.

\section{Resultados}

Las características de los participantes, según sospecha de deterioro cognitivo, se presentan en la Tabla 1. En resumen, personas con sospecha de deterioro cognitivo presentaron un mayor promedio de edad que los individuos clasificados como normales. La prevalencia de sospecha de deterioro cognitivo fue mayor en hombres que mujeres, en zonas urbanas que rurales, en población con bajos niveles de escolaridad (básica) e ingreso económico que en personas con niveles medios o altos. Estos también presentaron un menor peso corporal, IMC y PC que personas sin sospecha de deterioro cognitivo. Sin embargo, la prevalencia de obesidad y bajo peso fue más alta en personas con sospecha de deterioro cognitivo que en aquellos sin deterioro cognitivo. Los niveles 
Tabla 1. Características de la población según Mini-Mental (MMSE)

\begin{tabular}{|c|c|c|}
\hline & $\begin{array}{c}\text { Sin sospecha de deterioro } \\
\text { cognitivo }\end{array}$ & $\begin{array}{c}\text { Con sospecha de deterioro } \\
\text { cognitivo }\end{array}$ \\
\hline \multicolumn{3}{|l|}{ Sociodemográficas } \\
\hline Muestra encuestada, $\mathrm{n}^{*}$ & 1.215 & 169 \\
\hline Muestra expandida, $\mathrm{n}^{*}$ & 2.112 .031 & 280.733 \\
\hline $\begin{array}{l}\text { Sexo, }(\%) \\
\text { Hombres } \\
\text { Mujeres }\end{array}$ & $\begin{array}{l}44,6(39,1 ; 50,0) \\
55,4(49,9 ; 60,8)\end{array}$ & $\begin{array}{l}52,8(38.7 ; 66.5) \\
47,2(33.4 ; 61.2)\end{array}$ \\
\hline Edad (años) & $68,5(67,7 ; 69,2)$ & $76,3(73.8 ; 78.7)$ \\
\hline $\begin{array}{l}\text { Zona geográfica (\%) } \\
\text { Rural } \\
\text { Urbana }\end{array}$ & $\begin{array}{l}14,6(11,9 ; 17,7) \\
85,4(82,2 ; 88,0)\end{array}$ & $\begin{array}{l}29,2(16,1 ; 46,8) \\
70,8(53,1 ; 83,8)\end{array}$ \\
\hline $\begin{array}{l}\text { Nivel educacional (\%) } \\
\text { Básica } \\
\text { Media } \\
\text { Técnico-universitaria }\end{array}$ & $\begin{array}{l}46,9(41,8 ; 52,1) \\
37,8(32,5 ; 43,3) \\
15,3(11,0 ; 20,6)\end{array}$ & $\begin{array}{c}83,9(72,5 ; 91,2) \\
14,8(7,8 ; 26,2) \\
1,3(0,2 ; 4,8)\end{array}$ \\
\hline $\begin{array}{l}\text { Nivel de ingreso (\%) } \\
\text { Bajo } \\
\text { Medio } \\
\text { Alto }\end{array}$ & $\begin{array}{c}56,6(51,1 ; 61,9) \\
32,5(27,6 ; 37,8) \\
10,9(7,4 ; 156)\end{array}$ & $\begin{array}{c}82,7(69,4 ; 90,9) \\
17,3(9,0 ; 30,5) \\
0\end{array}$ \\
\hline Antropométricas & & \\
\hline Talla (m) & $1,58(1,57 ; 1.59)$ & $1,55(1,52 ; 1,56)$ \\
\hline Peso corporal (kg) & $71,8(704 ; 73.1)$ & $64,9(61,5 ; 68,2)$ \\
\hline $\operatorname{IMC}\left(\mathrm{kg} / \mathrm{m}^{2}\right)$ & $28,7(28,2 ; 29.3)$ & $27,1(25,8 ; 28,4)$ \\
\hline $\begin{array}{l}\text { Estado nutricional (\%) } \\
\text { Bajo peso } \\
\text { Normal } \\
\text { Sobrepeso } \\
\text { Obeso }\end{array}$ & $\begin{array}{c}7,7(5,6 ; 10,4) \\
34,9(29,6 ; 40,6) \\
29,6(25,1 ; 34,5) \\
27,8(23,3 ; 32,6)\end{array}$ & $\begin{array}{l}23,3(12,8 ; 38,1) \\
19,1(10,7 ; 31,6) \\
21,1(13,4 ; 31,7) \\
36,5(22,7 ; 52,9)\end{array}$ \\
\hline Perímetro de cintura $(\mathrm{cm})$ & $95,9(94.7 ; 97.1)$ & $93,1(89,7 ; 96,5)$ \\
\hline $\begin{array}{l}\text { Obesidad abdominal (\%) } \\
\text { Normal } \\
\text { Obeso central }\end{array}$ & $\begin{array}{l}46,7(41.4 ; 52.0) \\
53,3(47.9 ; 58.5)\end{array}$ & $\begin{array}{l}44,6(31,2 ; 59,9) \\
55,4(41,1 ; 68,8)\end{array}$ \\
\hline Estilo de vida & & \\
\hline Actividad física total (METs/h/semana) & $89,5(75,9 ; 103,1)$ & $47,6(22,1 ; 73, .2)$ \\
\hline Actividad física de transporte (min/día) & $38,1(31,8 ; 44,4)$ & $22,7(6,6 ; 38,9)$ \\
\hline Actividad física moderada (min/día) & $84,8(70,1 ; 99,5)$ & $23,9(12,2 ; 35,7)$ \\
\hline Actividad física vigorosa (min/día) & $34,4(23,2 ; 45,5)$ & $27,6(3,9 ; 51,3)$ \\
\hline $\begin{array}{l}\text { Prevalencia inactividad física (\%) } \\
\text { Activo } \\
\text { Inactivo }\end{array}$ & $\begin{array}{l}69,4(64,5 ; 73,9) \\
30,6(26,1 ; 35,4)\end{array}$ & $\begin{array}{l}37,1(24,8 ; 51,3) \\
62,9(48,6 ; 75,2)\end{array}$ \\
\hline Tiempo sedente (h/día) & $3,4(3,2 ; 3,7)$ & $4,3(3,1 ; 5,4)$ \\
\hline $\begin{array}{l}\text { Índice de Dieta Saludable (\%) } \\
\text { No saludable } \\
\text { Saludable } \\
\text { Muy saludable }\end{array}$ & $\begin{array}{l}32,6(28,1 ; 37,4) \\
40,9(35,6 ; 46,4) \\
26,5(21,9 ; 31,4)\end{array}$ & $\begin{array}{c}60,6(47,2 ; 72,4) \\
26,5(17,4 ; 38,0) \\
12,9(7,4 ; 21,4)\end{array}$ \\
\hline
\end{tabular}




\begin{tabular}{|c|c|c|}
\hline \multicolumn{3}{|l|}{ Horas de sueño (\%) } \\
\hline$<7 \mathrm{~h}$ & $48,3(43,1 ; 53,7)$ & $33,1(21,0 ; 47,8)$ \\
\hline $7-9 h$ & $35,3(30,2 ; 40,7)$ & $24,4(15,8 ; 35,8)$ \\
\hline$>9 h$ & $16,4(13,1 ; 20,0)$ & $42,5(28,3 ; 57,8)$ \\
\hline \multicolumn{3}{|l|}{ Tabaquismo (\%) } \\
\hline Nunca & $45,2(40,0 ; 50,5)$ & $55,1(39,8 ; 69,4)$ \\
\hline Exfumador & $35,1(29,9 ; 40,5)$ & $37,8(23,5 ; 54,5)$ \\
\hline Fumador & $19,7(15,8 ; 24,2)$ & $7,1(3,2 ; 14,9)$ \\
\hline \multicolumn{3}{|l|}{ Comorbilidad } \\
\hline \multicolumn{3}{|l|}{$\mathrm{DMT} 2(\%)$} \\
\hline No & $74,9(69,7 ; 79,3)$ & $67,2(49,1 ; 81,3)$ \\
\hline Si & $25,1(20,7 ; 30,2)$ & $32,8(18,6 ; 50,9)$ \\
\hline \multicolumn{3}{|c|}{ Hipertensión arterial (\%) } \\
\hline No & $33,8(28,7 ; 39,2)$ & $14,1(8,3 ; 22,9)$ \\
\hline $\mathrm{Si}$ & $66,2(60,7 ; 71,2)$ & $85,9(77,0 ; 91,8)$ \\
\hline \multicolumn{3}{|c|}{ Problemas de audición (\%) } \\
\hline No & $53,8(48,3 ; 59,1)$ & $23,9(15,5 ; 65,1)$ \\
\hline $\mathrm{Si}$ & $46,2(40,8 ; 51,6)$ & $76,1(64,8 ; 84,5)$ \\
\hline \multicolumn{3}{|l|}{ Depresión (\%) } \\
\hline No & $86,2(81,6 ; 89,7)$ & $78,0(63,8 ; 87,6)$ \\
\hline Si & $13,8(10,2 ; 18,3)$ & $22,0(12,3 ; 36,2)$ \\
\hline Puntaje mini mental & $17,1(16,9 ; 17,3)$ & $8,56(7,64 ; 9,48)$ \\
\hline
\end{tabular}

Datos presentados como promedio expandidos para variables continuas y como porcentaje expandidos para variables categóricas y sus respectivos 95\% IC. * el n-encuestado corresponde al número de individuos incluidos en la ENS 2009-2010, y el n-expandido indica a la cantidad de la población que representa a nivel nacional al utilizar los factores de expansión de la ENS 2009-2010.

de AF reportados fueron más bajos y el tiempo destinado a estar sentado más alto en personas con sospecha de deterioro cognitivo que sin deterioro cognitivo. Un 76,1\% de las personas con sospecha de deterioro cognitivo fueron clasificadas como físicamente inactivas en comparación a 46,2\% de las personas sin deterioro cognitivo. También se observó una mayor prevalencia de dieta no saludable, tabaquismo, DMT2, HTA, depresión y problemas de audición en población con sospecha de deterior cognitivo en comparación a la clasificada sin sospecha (Tabla 1 ).

La Tabla 2 muestra la asociación entre sospecha de deterioro cognitivo según tertiles de AF. Los resultados no ajustados (Modelo 0 ) revelaron que en comparación al grupo que realiza $<48 \mathrm{~min}$ de AF por día, aquellos con niveles moderados (48-248 $\mathrm{min} /$ día) y altos (> $248 \mathrm{~min} /$ día) de AF presentan una asociación negativa con sospecha de deterioro cognitivo. Al ajustar por las variables de confusión (Modelo 1, 2 y 3) los resultados disminuyeron la magnitud de la asociación, pero permanecieron significativos. Para el modelo más ajustado (Modelo 3) niveles moderados o altos de AF se asociaron con un odds ratio de 0,57 (95\%IC $[0,33 ; 0,82], \mathrm{p}=0,007)$ y $0,58(95 \%$ IC $[0,32 ; 0,83]$, $\mathrm{p}=0,009)$ para sospecha de deterioro cognitivo, respectivamente (Figura 1 ).

La Tabla 3 muestra la asociación entre sospecha de deterioro cognitivo según tertiles de tiempo sedente. Los análisis no ajustados indican que personas con niveles altos (4-8 h al día) o muy altos ( $>8 \mathrm{~h}$ al día) presentan una asociación positiva con sospecha de deterioro cognitivo en comparación a aquellos que reportaron $<4 \mathrm{~h}$ /día. $\mathrm{Al}$ ajustar los análisis por factores de confusión (Modelo más ajustado, Modelo 3) la asociación con niveles altos de tiempo sedente no fue significativa. Sin embargo, la asociación con niveles muy altos de tiempo sedente se mantuvo significativamente asociada a sospecha de deterioro cognitivo mostrando incluso un incremento en la magnitud de la asociación (OR: 3,70 [95\% IC: 1,37 a 6,03], $\mathrm{p}=0,010)$ (Figura 2 y Tabla 3 ). 
Tabla 2. Asociación entre tertiles de actividad física y sospecha de deterioro cognitivo en población adulta mayor en Chile

\begin{tabular}{|c|c|c|c|c|c|c|c|}
\hline & $\begin{array}{c}\text { Bajo } \\
\text { (< } 48 \mathrm{~min} / \text { día) } \\
\text { OR }\end{array}$ & $\begin{array}{c}\text { Medio } \\
\text { (48-248 min/día) } \\
\text { OR (95\% IC) }\end{array}$ & Valor p & $\begin{array}{c}\text { Alto } \\
\text { (> } 248 \mathrm{~min} / \text { día }) \\
\text { OR }(95 \% \text { IC })\end{array}$ & Valor p & $\begin{array}{l}\text { Tendencia } \\
\text { OR (95\% IC) }\end{array}$ & Valor p \\
\hline Modelo 0 & 1,00 (Ref.) & $0,39(0,26 ; 0,52)$ & $<0,0001$ & $0,32(0,21 ; 0,43)$ & $<0,0001$ & $0,53(0,42 ; 0,64)$ & $<0,0001$ \\
\hline Modelo 1 & 1,00 (Ref.) & $0,42(0,27 ; 0,57)$ & $<0,0001$ & $0,41(0,25 ; 0,57)$ & $<0,0001$ & $0,60(0,47 ; 0,73)$ & $<0,0001$ \\
\hline Modelo 2 & 1,00 (Ref.) & $0,48(0,29 ; 0,67)$ & 0,004 & $0,53(0,31 ; 0,75)$ & 0,006 & $0,70(0,53 ; 0,86)$ & 0,008 \\
\hline Modelo 3 & 1,00 (Ref.) & $0,57(0,33 ; 0,82)$ & 0,007 & $0,58(0,32 ; 0,83)$ & 0,009 & $0,74(0,55 ; 0,92)$ & 0,044 \\
\hline
\end{tabular}

Datos presentados como odds ratio expandidos y sus respectivos intervalos de confianza (OR (95\% IC). El grupo de referencia utilizado corresponde al tertil más bajo de $\operatorname{AF}(<48 \mathrm{~min} /$ día). El odds ratio para tendencia indica el cambio en OR equivalente a incrementar en un tertil los niveles de AF. Los análisis fueron ajustados progresivamente como se indica a continuación: Modelo 0 no se ajustó por variables confundidoras; Modelo 1 fue ajustado por edad, sexo, región, zona geográfica de residencia, nivel educacional e ingreso económico; Modelo 2 fue ajustado por las variables del modelo 1 más IMC, tabaquismo, índice de alimentación saludable, horas destinadas a dormir, tiempo destinado a estar sentado; y el Modelo 3 fue ajustado por las variables del modelo 2 más DMT2, HTA, depresión y problemas auditivos.

Tabla 3. Asociación entre tertiles de tiempo destinado a estar sentado y sospecha de deterioro cognitivo en población adulta mayor en Chile

\begin{tabular}{|c|c|c|c|c|c|c|c|}
\hline & $\begin{array}{c}\text { Bajo } \\
\text { (< } 4 \text { h/día) } \\
\text { OR }\end{array}$ & $\begin{array}{c}\text { Alto } \\
\text { (4-8 h/día) } \\
\text { OR (95\% IC) }\end{array}$ & Valor p & $\begin{array}{l}\text { Muy Alto } \\
\text { (> } 8 \text { h/día) } \\
\text { OR (95\% IC) }\end{array}$ & Valor p & $\begin{array}{c}\text { Tendencia } \\
\text { OR (95\% IC) }\end{array}$ & Valor $p$ \\
\hline Modelo 0 & 1,00 (Ref.) & $1,70(0,79 ; 2,60)$ & 0,174 & $3,27(1,46 ; 5,08)$ & 0,004 & $1,79(1,19 ; 2,40)$ & 0,005 \\
\hline Modelo 1 & 1,00 (Ref.) & $1,31(0,61 ; 2,02)$ & 0,485 & $3,81(1,68 ; 5,93)$ & 0,001 & $1,85(1,22 ; 2,48)$ & 0,004 \\
\hline Modelo 2 & 1,00 (Ref.) & $1,67(0,77 ; 2,58)$ & 0,190 & $3,10(1,27 ; 4,93)$ & 0,013 & $1,75(112 ; 2,37)$ & 0,014 \\
\hline Modelo 3 & 1,00 (Ref.) & $1,55(0,67 ; 2,42)$ & 0,297 & $3,70(1,37 ; 6,03)$ & 0,010 & $1,85(1,12 ; 2,57)$ & 0,016 \\
\hline
\end{tabular}

Datos presentados como odds ratio expandidos y sus respectivos intervalos de confianza (OR (95\% IC)). El grupo de referencia utilizado corresponde al tertil más bajo de tiempo destinado a estar sentado $(<4 \mathrm{~h} /$ día). El odds ratio para tendencia indica el cambio en OR equivalente a incrementar en un tertil los niveles de tiempo destinado a estar sentado. Los análisis fueron ajustados progresivamente como se indica a continuación: Modelo 0 no se ajustó por variables confundidoras; Modelo 1 fue ajustado por edad, sexo, región, zona geográfica de residencia, nivel educacional e ingreso económico; Modelo 2 fue ajustado por las variables del modelo 1 más IMC, tabaquismo, índice de alimentación saludable, AF, tiempo destinado a dormir; y el Modelo 3 fue ajustado por las variables del modelo 2 más DMT2, HTA, depresión y problemas auditivos.

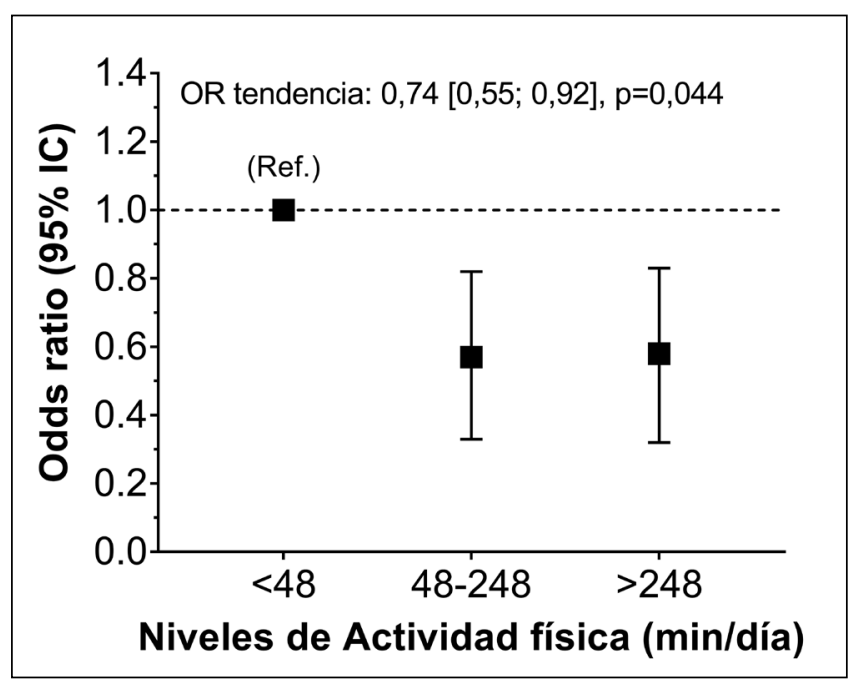

Figura 1. Odds ratio para sospecha de deterioro cognitivo según niveles de actividad física en población adulta mayor en Chile. Datos presentados como odds ratio expandidos y sus respectivos intervalos de confianza (OR $(95 \%, I C)$ ). El grupo de referencia utilizado corresponde al tertil más bajo de niveles de actividad física ( $<48 \mathrm{~min} /$ día). El odds ratio para tendencia indica el cambio en OR equivalente a incrementar en un tertil los niveles de AF. Los análisis fueron ajustados progresivamente por edad, sexo, región, zona geográfica de residencia, nivel educacional, ingreso económico, IMC, tabaquismo, índice de alimentación saludable, tiempo destinado a estar sentado, tiempo destinado a dormir, DMT2, HTA, depresión y problemas auditivos. 


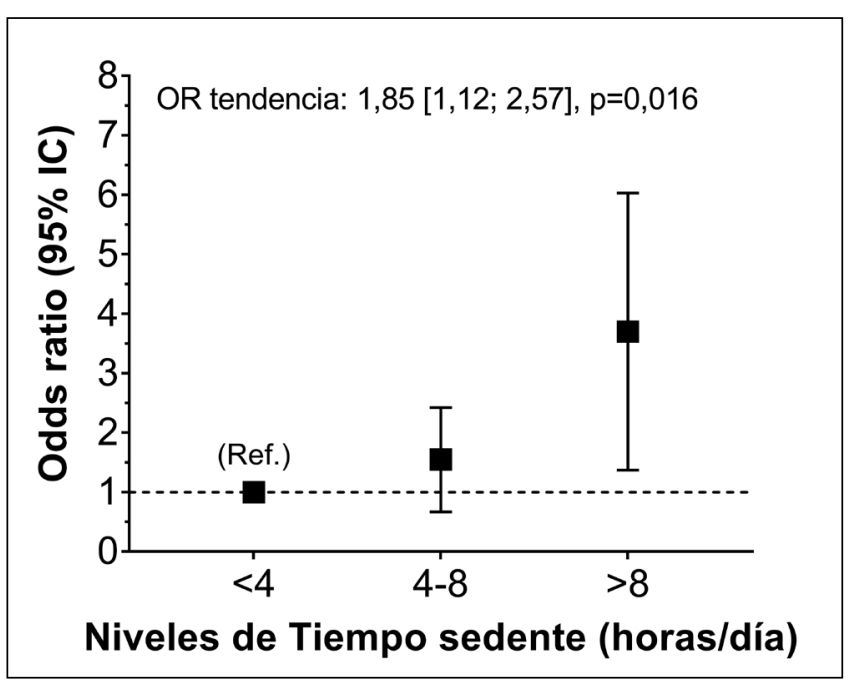

Figura 2. Odds ratio para sospecha de deterioro cognitivo según niveles de tiempo destinado a estar sentado en población adulta mayor en Chile. Datos presentados como odds ratio expandidos y sus respectivos intervalos de confianza (OR $(95 \% \mathrm{IC})$ ). El grupo de referencia utilizado corresponde al tertil más bajo de tiempo sedente $(<4 \mathrm{~h} /$ día). El odds ratio para tendencia indica el cambio en OR equivalente a incrementar en un tertil los niveles de tiempo destinado a estar sentado. Los análisis fueron ajustados progresivamente por edad, sexo, región, zona geográfica de residencia, nivel educacional, ingreso económico, IMC, tabaquismo, índice de alimentación saludable, AF, tiempo destinado a dormir, DMT2, HTA, depresión y problemas auditivos.

\section{Discusión}

Los principales resultados de este estudio señalan que altos niveles de AF se asocian a sospecha de deterioro cognitivo mientras que altos niveles de tiempo destinado a estar sentado se asocian inversamente a sospecha de deterioro cognitivo. Es importante destacar que esta asociación fue independiente de factores de confusión incluyendo características sociodemográficas, obesidad, estilos de vida y multimorbilidad. Cabe destacar que la asociación de AF y tiempo sedente con sospecha de deterioro cognitivo fue mutuamente independiente, lo cual indica que independiente del tiempo destinado a realizar AF las personas adultas mayores que destinan más de $8 \mathrm{~h}$ aún presentan una asociación con sospecha de deterioro cognitivo, y viceversa. La importancia de estos resultados para salud publica radican en la incorporación de recomendaciones que sugieran incrementar los niveles de AF en la población, pero que también sugieran disminuir el tiempo que la población adulta mayor destina a estar sentada.

Si bien existen estudios que han investigado el efecto de intervenciones basadas en actividad física sobre el deterioro cognitivo, la evidencia disponible a la fecha es insuficiente para determinar el rol de la actividad física sobre la prevención e deterioro cognitivo ${ }^{23}$. Una revisión sistemática publicada recientemente por Brasure y cols. ${ }^{23}$, la cual incluyo 32 estudios de intervención, reporto que se requiere mayor cantidad de evidencia, y que los estudios actuales son de bajo poder estadístico $\mathrm{y}$ con un alto nivel de heterogeneidad y diversidad en la metodología utilizada para determinar deterioro cognitivo ${ }^{23}$. A la fecha solo una intervención de carácter multicomponente (dieta y actividad física) ha mostrado un efecto moderado sobre deterioro cognitivo, sin embargo, esta intervención fue basada en más de un estilo de vida, por lo que es imposible evaluar el efecto aislado de la actividad física sobre deterioro cognitivo ${ }^{24}$.

En relación a los potenciales mecanismos que podrían explicar esta asociación entre AF, tiempo sedente y deterioro cognitivo se encuentran diversos estudios experimentales los cuales han indicado que el incremento de la capacidad aeróbica aumenta el flujo sanguíneo cerebral, mejorando la utilización del oxígeno y la glucosa del cerebro, así como el incremento de la insulina, estimulando la neurogénesis y aumentando las interconexiones sinápticas ${ }^{25}$. Asimismo, la $\mathrm{AF}$ favorece la regulación de neurotransmisores y la estimulación de liberación de calcio. Todos ellos son necesarios para mantener el funcionamiento neuronal, promover un estado de ánimo positivo y mejorar la función cognitiva ${ }^{26}$. Además, el ejercicio puede incrementar la capacidad de reserva cognitiva del cerebro, reduce la tasa de envejecimiento y disminuye el riesgo de desarrollo de enfermedades neurodegenerativas. Las personas que son activas 
durante toda la vida, especialmente durante la mediana edad, se ha reportado que presentan un mejor desarrollo de las funciones cognitivas durante más tiempo ${ }^{27}$. No obstante, también se indica que la $\mathrm{AF}$, es efectiva en promover un mayor contacto social, y que como resultado de esto mismo, puede estimular a que nuestro cerebro mantenga su funcionamiento cognitivo elevado y mantenido en el tiempo ${ }^{28}$.

Si bien este estudio presenta diferentes fortalezas, como lo es la representatividad nacional, utilización de métodos estandarizados de evaluación, y medición de factores o variables de confusión, las cuales fueron integradas en nuestros análisis, este estudio no está exento de limitaciones que deben ser consideradas al momento de interpretar los resultados e implicaciones. Debido a su diseño de corte transversal no podemos determinar una asociación causal de AF y tiempo sedente con deterioro cognitivo. Si bien el instrumento utilizado no es un gold estándar para deterioro cognitivo, y presenta un moderado nivel de sensibilidad y especificidad $^{29}$, es una prueba ampliamente utilizada en estudios clínicos para determinar sospecha de deterioro cognitivo. Otra limitante, es que, si bien ajustamos nuestros análisis por una amplia cantidad de factores de confusión, no podemos descartar que las asociaciones reportadas pudieran ser atribuidas a factores no medidos o a cambios experimentados en AF y tiempo sedente en edades tempranas y anterior a la aplicación de la ENS 2009-2010. El auto-reporte de AF y tiempo sedente tienden a ser sobreestimados y subestimados, respectivamente, lo cual podría haber influido al estimar la verdadera magnitud de la asociación entre estos factores de exposición y sospecha de deterioro cognitivo.

\section{Conclusión}

Los resultados del presente estudio indican que altos niveles de AF se asocian negativamente con sospecha de deterioro cognitivo mientras que el tiempo destinado a estar sentado se asocia positivamente con sospecha de deterioro cognitivo. Dichos resultados podrían informar la creación de futuras guías de AF para la población adulta mayor en Chile, las cuales promuevan la practica regular de AF como también así una reducción el tiempo destinado a estar sentado.

\section{Referencias}

1. WHO. Global Health and Aging 2011. World Health Organization; US National Institute of Aging, 2011. October 2011. Report No.: Contract No.: 06 June. Available: http://www.who.int/ageing/publications/ global_health.pdf.

2. WHO. Dementia http://www.who.int: World Health Organization; 2017. Available: http://www.who.int/ mediacentre/factsheets/fs362/en/.

3. Hung WW, Ross JS, Boockvar KS, Siu AL. Recent trends in chronic disease, impairment and disability among older adults in the United States. BMC Geriatr 2011; 11 (1): 47. doi:

4. Wolff JL, Starfield B, Anderson G. Prevalence, expenditures, and complications of multiple chronic conditions in the elderly. Arch Intern Med 2002; 162 (20): 2269-76. doi:10.1186/1471-2318-11-47.

5. Livingston G, Sommerlad A, Orgeta V, Costafreda SG, Huntley J, Ames D, et al. Dementia prevention, intervention, and care. Lancet 2017; 390 (10113): 2673-734. doi: 10.1016/S0140-6736(17)31363-6.

6. Murman DL, editor The impact of age on cognition. Seminars in hearing; 2015: Thieme Medical Publishers.

7. Blondell SJ, Hammersley-Mather R, Veerman JL. Does physical activity prevent cognitive decline and dementia?: A systematic review and meta-analysis of longitudinal studies. BMC Public Health 2014; 14: 510. doi: 10.1186/1471-2458-14-510.

8. Hamer M, Stamatakis E. Prospective study of sedentary behavior, risk of depression, and cognitive impairment. Med Sci Sports Exerc 2014; 46 (4): 718. doi:

9. Edwards MK, Loprinzi PD. The association between sedentary behavior and cognitive function among older adults may be attenuated with adequate physical activity. J Phys Act Health 2017; 14 (1): 52-8. doi: 10.1249/ MSS.0000000000000156.

10. Wikee G, Martella D. Capacidad física y reserva cognitiva como factores protectores de las funciones atencionales en adultos mayores. Rev Med Chile 2018; 146 (5): 570-7.

11. García-Hermoso A, Ramírez-Velez R, Celis-Morales CA, Olloquequi J, Izquierdo M. Can physical activity attenuate the negative association between sitting time and cognitive function among older adults? A mediation analysis. Exp Geronto. 2018;106:173-7. doi: 10.1016/j. exger.2018.03.002.

12. Celis-Morales C, Salas C, Alduhishy A, Sanzana R, Martinez MA, Leiva A, et al. Socio-demographic patterns of physical activity and sedentary behaviour in Chile: results from the National Health Survey 2009- 
2010. J Public Health (Oxf) 2016; 38 (2): e98-e105. doi: 10.1093/pubmed/fdv079.

13. Garrido-Méndez A, Concha-Cisternas Y, Petermann-Rocha F, Díaz-Martínez X, Leiva AM, Troncoso $\mathrm{C}$, et al. Influencia de la edad sobre el cumplimiento de las recomendaciones de actividad física: Resultados de la Encuesta Nacional de Salud en Chile 2009-2010. Rev Chil Nutr 2019; 46: 121-8. doi: 10.4067/S071775182019000200121.

14. OMS. Informe mundial sobre el envejecimiento y la salud. Organizacion Mundial de ls Salud, Ginebra; 2015.

15. OMS. Encuesta nacional de Salud 2009-2010. Ministerio de Salud de Chile; 2010.

16. Burns A, Brayne C, Folstein M. Mini-Mental State: A practical method for grading the cognitive state of patients for the clinician. J Psychiatr Res 1975; 12: 189-98.

17. Quiroga P, Albala C, Klaasen G. Validation of a screening test for age associated cognitive impairment, in Chile. Rev Med Chile 2004; 132 (4): 467-78. doi: 10.1016/j.nrleng.2018.12.008.

18. OPS. Parte 1: Módulos de Valoración clínica. Módulo 5: Valoración Nutricional del Adulto Mayor. Organización Panamericana de la Salud. 2003.

19. Organization WH. Obesity: Preventing and Managing the Global Epidemic (2000). 894 WHO: Geneva. WHO Technical Report Series Ref Type: Report. 2000:http:// www.who.int/nutrition/publications/obesity/WHO_ TRS_894/en/.doi:

20. Questionnaire WGPA. GPAQ version 2.0. World Health Organization. 2009.

21. Dussaillant C, Echeverría G, Villarroel L, Yu C, Rigotti A, Marín P. Metabolic syndrome prevalence is not associated with diet quality in the Chilean elderly population: a cross sectional analysis from the National Health Survey 2009-2010. J Aging Res Clin Practice 2016; 5 (3): 132-8. doi: dx.doi.org/10.14283/ jarcp.2016.104

22. Dussaillant C, Echeverria G, Villarroel L, Marín P, Rigotti A. Unhealthy food intake is linked to higher prevalence of metabolic syndrome in Chilean adult population: cross sectional study in 2009-2010 National Health Survey. Nutr Hosp 2015; 32 (5): 2098-104. doi: 10.3305/nh.2015.32.5.9657.

23. Brasure M, Desai P, Davila H, Nelson VA, Calvert C, Jutkowitz E, et al. Physical Activity Interventions in Preventing Cognitive Decline and Alzheimer-Type Dementia: A Systematic ReviewPhysical Activity to Prevent Cognitive Decline and Dementia. Ann Intern Med 2018; 168 (1): 30-8. doi: 10.7326/M17-1528.

24. Ngandu T, Lehtisalo J, Solomon A, Levälahti E, Ahtiluoto S, Antikainen R, et al. A 2 year multidomain intervention of diet, exercise, cognitive training, and vascular risk monitoring versus control to prevent cognitive decline in at-risk elderly people (FINGER): a randomised controlled trial. Lancet 2015; 385 (9984): 2255-63. doi: 10.1016/S0140-6736(15)60461-5.

25. Archer T. Physical exercise alleviates debilities of normal aging and Alzheimer's disease. Acta Neurol Scand 2011; 123 (4): 221-38. doi: 10.1111/j.1600-0404.2010.01412.x.

26. Tseng C-N, Gau B-S, Lou M-F. The effectiveness of exercise on improving cognitive function in older people: a systematic review. J Nurs Res 2011; 19 (2): 119-31. doi: 10.1097/JNR.0b013e3182198837.

27. Sofi F, Valecchi D, Bacci D, Abbate R, Gensini GF, Casini A, et al. Physical activity and risk of cognitive decline: a meta-analysis of prospective studies. J Intern Med 2011; 269 (1): 107-17. doi: 10.1111/j.13652796.2010.02281.x.

28. Kulmala J, Solomon A, Kareholt I, Ngandu T, Rantanen T, Laatikainen T, et al. Association between mid- to late life physical fitness and dementia: evidence from the CAIDE study. J Intern Med 2014; 276 (3): 296-307. doi: 10.1111/joim.12202.

29. Carnero Pardo C, Cruz Orduña I, Espejo Martínez B, Cárdenas Viedma S, Torrero García P, Olazarán Rodríguez J. Efectividad del Mini-Mental en la detección del deterioro cognitivo en Atención Primaria. Aten Primaria 2013; 45 (8): 426-33. doi: 10.1016/j.aprim.2013.04.009. 